\title{
VIDEOJUEGOS, TELEVISIÓN Y RENDIMIENTO ACADÉMICO EN ALUMNOS DE PRIMARIA.
}

\section{VIDEO GAMES, TELEVISION AND ACADEMIC PERFORMANCE IN ELEMENTARY SCHOOL STUDENTS}

\author{
Dra. M.del Mar Badia Martín ${ }^{1}$ \\ mar.badia@uab.es \\ Dra. Mercè ClarianaMuntada ${ }^{1}$ \\ merce.clariana@uab.cat \\ Dra. Concepción Gotzens Busquets² \\ c.gotzens@uib.es \\ Dr. Ramon Cladellas Pros ${ }^{1}$ \\ Ramon.Cladellas@uab.cat \\ Dra.Teresa Dezcallar Sáez ${ }^{1}$ \\ Teresa.Dezcallar@uab.cat
}

\begin{abstract}
${ }^{1}$ Universidad Autónoma de Barcelona. Facultad de Psicología.Departamento de Psicología Básica, Evolutiva y de la Educación. Edificio B. Campus de la UAB. 08193,Bellaterra (Cerdanyola del Vallès), Barcelona (España)

${ }^{2}$ Universidad de las Islas Baleares (España). Departamento de Pedagogía Aplicada y Psicología de la Educación. Edificio Guillem Cifre de Catalunya. Cra.deValldemosa, Km7.5. 07122, Palma. Islas Baleares (España)
\end{abstract}

El mal uso de las tecnologías puede repercutir en un menor rendimiento académico. En el presente trabajo se ha analizado la relación entre el tiempo dedicado a la televisión, uso de videojuegos, y las notas de los alumnos, en una muestra de educación primaria de Cataluña y de las Islas Baleares, participando 711 alumnos, entre 6 y 13 años. Los resultados muestran que el número de horas de televisión puede guardar una relación negativa con el rendimiento en matemáticas. Se concluye que el uso correcto de videojuegos puede ser beneficioso en áreas escolares como las matemáticas, no así la televisión.

Palabras clave:Rendimiento académico, televisión, videojuegos, alumnos, educación primaria.

The bad use of the technology can generate a lower academic performance. In this paper the relationship between time spent on television and video games and the grades obtained by a sample of primary education students in Catalonia and the Balearic Islands is analyzed. The study evaluates 711 students, from 6 to 13 years old. The study shows that the number of hours watching television has a negative relationship with the performance in Mathematics, but not the use of video games. In conclusion, the use of video games can be beneficial in Mathematics.

Keywords:Academic achievement, television, video-games, students, primary education.

Píxel-Bit. Revista de Medios y Educación. No 46. Enero 2015. ISSN: 1133-8482. e-ISSN: 2171-7966. doi: http://dx.doi.org/10.12795/pixelbit.2015.i46.02 


\section{Introducción.}

La mayor parte de los estudios sobre los efectos de la televisión en los niños se han enfocado estudiando variables tales como la obesidad, la inactividad, el problema atencional, los problemas de trastornos del sueño, la agresión y el alto riesgo de mal comportamiento (Singh \& Gaurav, 2013; Rodríguez, 2005). Por otro lado, las investigaciones sobre el uso de videojuegos también han tenido en cuenta estos factores, pero han puesto mayor énfasis en analizar no sólo los problemas que el exceso de su uso conlleva, sino también los beneficios en el rendimiento académico (Sedeño, 2010). Así pues, y a diferencia de lo que sucede con la televisión, algunos profesionales e investigadores educativos (p. ej., Green \& Bavelier, 2003; Trick, Jaspers-Fayer \& Sethi, 2005), atribuyen al uso de videojuegos una mejora de los alumnos en ciertas áreas, si bien Villadangos y Labrador (2009), comentan que la implantación de los videojuegos en la sociedad no está exenta de polémica, encontrando defensores y detractores de su uso.

Con todo, son escasos los estudios que han investigado la relación entre rendimiento académico y horas de uso de pantallas, entendiéndose éstas como videojuegos y televisión.Ante este panorama consideramos importante abordar este tema.

\section{Videojuegos y rendimiento.}

Diferentes estudios con niños y adolescentes (Castells \& Bofarull, 2002; Bringas, Rodríguez \& Herrero, 2008), demuestran que el rendimiento escolar puede verse afectado, no solamente por el tiempo que dedican al estudio diariamente, el nivel de inteligencia y/o la responsabilidad que presentan, sino también por las diferentes actividades que los estudiantes realizan en su vida diaria, tales como el consumo de videojuegos.

Otras investigaciones como la de Vallejos y Capa (2010), han revelado la existencia de un patrón de mala adaptación de uso de videojuegos cuyos síntomas son muy similares a los de las adicciones.

Los hábitos y comportamientos de los alumnos influyen en su estado de salud y en su rendimiento académico. Es posible que determinados hábitos, como el no dormir el número de horas requerido según la edad y la falta de estudio fuera del horario de clases, entre otros, pueda causar problemas escolares, como indican Aguilar, Cumbá, Cortés, Collado, García y Pérez (2010). Un estudio de estos autores puso de manifiesto que los alumnos del grupo «con bajo rendimiento académico» presentaban un porcentaje muy superior, en relación con el grupo «sin bajo rendimiento académico», en las variables: no juega al aire libre, estudia tiempo insuficiente, no lee y juega con videojuegos, entre otros aspectos. Centrándonos en el uso de los videojuegos, cabe destacar que la motivación de la mayoría de investigaciones ha focalizado su interés en demostrar los efectos, ya sean positivos o negativos, que producen en la población.

Los escasos estudios longitudinales que abordan la investigación sobre los videojuegos y el rendimiento encuentran una asociación entre el uso problemático de videojuegos y bajo rendimiento escolar. $\mathrm{Si}$ bien, niveles moderados de juego no se asocian con un bajo rendimiento escolar (Ferguson, 2011), incluso, podrían relacionarse con un mejor rendimiento (Llorca, Bueno, Villar \& Díez, 2010).

Una parte de la comunidad científica considera que su mal uso, podría empeorar el 
aprendizaje de los alumnos, ya que contribuiría a aumentar su distracción. Esto iría en detrimento del provecho asociado a los deberes, lecturas y realización de actividades lúdicas al aire libre, lo que no es obstáculo para que algunos investigadores consideren que un buen uso de los videojuegos podría mejorar algunas áreas de conocimiento. Las investigaciones a nivel del estado español que se han interesado por los videojuegos han tenido en cuenta sus implicaciones a nivel de consumo, su relación con la autoestima, y cómo afectan éstos en general a la conducta (Marquès, 2000; Rodríguez, 2002; Gros, 2007; Etxeberria, 2008), pero no se han centrado en estudiar si el uso de esta pantalla tiene efectos beneficiosos en el ámbito académico.

En 1992, la preferencia de los niños por los videojuegos era ya muy alta, llegando a superar el $40 \%$ de los juegos que se vendieron. El videojuego ocupaba el primer lugar en la elección de juegos del $62 \%$ de los niños españoles, situándose en segundo lugar en el caso de las niñas. Si bien este crecimiento progresivo parece haberse estancado, los juegos con pantallas siguen siendo uno de los objetos preferidos por niños y adolescentes (Etxeberria, 1999).

Como comentábamos anteriormente, en las últimas décadas han surgido detractores y partidarios de este tipo de juegos. Los primeros consideran que producen aislamiento $\mathrm{y}$ adicción, que pueden llegar a fomentar conductas violentas en función de los juegos que se utilicen, que limitan la imaginación afectando al rendimiento académico, y los más extremos hablan de los efectos nocivos sobre la salud, considerando que son causantes de ataques epilépticos y de pérdida de visión. En esta línea Bailey, West, y Anderson (2010) afirman que jugar en exceso reduce la capacidad de atención en actividades como leer, estudiar o atender en clase.

Por otro lado, los partidarios de su uso, perciben en ellos un importante potencial educativo ya que estimulan la capacidad lógica, el razonamiento espacial, el desarrollo de estrategias encaminadas a la resolución de problemas, la atención y observación, la concentración, la reflexión, la creatividad e imaginación, entre otros aspectos (Rodríguez, 2002; Marks, 1985; Gagnon; 1985; Silvern, 1986; Greenfield, 1994; Ricci, 1994; Marquès, 2000).

Según Roschelle, Pea, Hoadley, Gordin y Means (2000), cabe la posibilidad que las ventajas que se obtengan de su uso no sean fácilmente cuantificables mediante las pruebas al uso, sobre todo en el caso de materias específicas evaluadas en un tiempo y contexto determinado. Pero es probable que en el ámbito educativo faciliten el desarrollo de habilidades de orden superior, como la selección y asociación de datos.

Como hemos dicho, si bien las investigaciones no son definitivas, la mayoría de ellas indican que muchos videojuegos, sobre todo los educativos, favorecen el desarrollo de determinadas habilidades, como por ejemplo la atención, concentración espacial, resolución de problemas, creatividad, etc., por lo que se concluye que en su conjunto, desde el punto de vista cognitivo, éstos supone algún tipo de ayuda en el desarrollo intelectual (White, 1984; Mandinacht, 1987; Okagaki \& Frensch, 1994). Asimismo, se sugiere que quienes utilizan videojuegos adquieren mejores estrategias de conocimiento, modos de resolver problemas, se benefician en sus habilidades espaciales y aumenta su precisión y capacidad de reacción (McFarlane, 2002). Frente a ello, no se dispone de evidencias de los efectos contrarios, aunque no debemos 
olvidar que es importante regular el número de horas invertidas en jugar, en lugar de invertirlas en leer o realizar actividades lúdicas de otro tipo.

\section{La televisión y su relación con el apren- dizaje.}

Si bien los videojuegos son la actividad estrella para muchos niños y adolescentes, no podemos olvidar la televisión, que forma parte de la mayoría de hogares desde hace décadas.La televisión es un arma poderosa que ha desplazado, en muchos casos, a la lectura y a otras actividades lúdicas. La cultura de la imagen parece haber desplazado a la cultura escrita, con las consecuencias que ello tiene para la educación. Ver la televisión no requiere apenas ninguna habilidad, ni tampoco, a diferencia de los videojuegos, se ha averiguado que ayude a desarrollar ninguna (Marquès, 2000). No obstante, parece oportuno señalar que no es mala en sí misma, sino en el uso que se le dé. El aspecto perjudicial de este medio se asocia principalmente a la falta de organización del tiempo que se le dedica y a la inadecuación en la selección de los programas. Al contrario de lo que ocurre cuando leemos o mantenemos una conversación, cuando vemos la televisión, nos exponemos a un movimiento incesante de imágenes, por lo que la rapidez con la que nos vemos obligados a absorber la información, nos convierte en receptores pasivos de la misma.

Se ha escrito mucho sobre la televisión y su relación con la violencia y agresividad en la población infantil y juvenil (Clemente \& Vidal, 1996; Donnerstein, 2004; Garrido, 2004) y sobre los modelos que ésta les aporta (Álvarez \& López, 2007; López, González \& Medina, 2011) y también sobre las horas que los niños y jóvenes pasan delante de estas pantallas (Villadangos \& Labrador, 2009). Sin embargo, disponemos de escasa literatura que relacione si, un uso excesivo, tiene algún efecto sobre el rendimiento académico, y más concretamente sobre la lengua y las matemáticas como asignaturas de la educación primaria.

En el estudio de Mark, Boyce y Janssen (2006), sobre el uso de pantallas en jóvenes canadienses, se vio que, sólo el 18\% de las niñas y el 14\% de los niños cumplían la recomendación de dedicar no más de dos horas diarias a las pantallas, especialmente a la televisión.

No podemos dejar de señalar el estudio de Ulpiano (1995), en el que se realizó un análisis descriptivo sobre el tiempo que dedicaban los alumnos de primaria a ver la televisión y su posible relación con el rendimiento académico. Los resultados coincidieron con la literatura revisada, mostrando que el promedio de ver la televisión era de unas 3 horas y media al día, siendo los alumnos de género masculino los que más tiempo pasaban delante de ella. Todas las variables de rendimiento presentaron una correlación negativa respecto a la variable televisión. Es decir, a mayor número de horas dedicadas a la televisión, menor era el rendimiento escolar en todas las materias. En esta misma línea, investigaciones como la de Kaiser Family Foundation (2003), han demostrado que los niños que ven demasiada televisión desde pequeños, pueden llegar a tener problemas en la adquisición del lenguaje y también en el desarrollo y adquisición de la escritura.

Recientemente, se ha comprobado que cada hora adicional de exposición a la televisión, por encima de las dos horas máximas recomendadas por la Academia Americana de Pediatría para niños mayores de 2 años, reper- 
cute, posteriormente, en una menor implicación en asignaturas como matemáticas, así como estilos de vida más sedentarios y peores hábitos nutricionales (Pagani, Fitzpatrick, Barnett \& Dubow (2010)).

En definitiva, buena parte de los estudios realizados indican que el tiempo invertido para ver la televisión impide a los niños que se puedan dedicar a otras actividades enriquecedoras que les permitan fomentar el desarrollo cognitivo, conductual y motor.

\section{Objetivos de la investigación.}

El objetivo de esta investigación es analizar las posibles relaciones entre tiempo de exposición a la televisión y uso de videojuegos con el rendimiento académico de alumnos de educación primaria en las áreas de matemáticas y lengua, dado que son las áreas comúnmente medidas cuando se trata de valorar el aprovechamiento en la escuela.

\section{Método.}

\subsection{Sujetos.}

Un total de 711 alumnos de primero a sexto de primaria, 359 niños $(50.5 \%)$ y 352 niñas $(49.5 \%)$ con edades comprendidas entre los 6 y los 13 años, participaron en el estudio. Provenían de escuelas públicas y privadas de la provincia de Barcelona (43\% de la muestra) y de la provincia de las Islas Baleares (57\%). La distribución de niños y niñas está homogéneamente repartida entre las escuelas barcelonesas y mallorquinas, $\mathrm{x}^{2}(1, n=$ $711)=1.28, p=.26$.

\subsection{Instrumentos y variables.}

Se elaboró un cuestionario para padres ex profeso para el estudio, mediante el cual se solicitaron datos sobre variables socioeconómicas de las familias, así como las calificaciones escolares de los alumnos en una escala de 0 (nota más baja) a 10 (nota más alta), y finalmente se preguntó el tiempo que el alumno miraba la televisión a la semana y también el que dedicaba a jugar con juegos de pantallas o videojuegos. Más concretamente, las variables analizadas en este estudio son:

- Género. Variable dicotómica que indica el género del alumno con dos categorías: Masculino / Femenino.

- Mat. Notas de matemáticas de la última evaluación. Se tomaron como una variable continua de 0 a 10 puntos o también como una variable dicotómica de dos categorías: Suspenso / Aprobado.

- Len. Notas de lenguaje de la última evaluación. Igual que la anterior, se tomaron como una variable continua de 0 a 10 puntos o también como una variable dicotómica de dos categorías: Suspenso / Aprobado.

- TV. Variable continua que indica los minutos a la semana que los alumnos miran la televisión.

- VJ. Variable continua que indica los minutos a la semana que los niños dedican a los juegos de pantallas.

\subsection{Procedimiento.}

Teniendo en cuenta que las informaciones facilitadas por los padres resultan ciertamente de gran utilidad (Bode \& Hirner, 2013; Gawrilow, Morgenroth, Schultz, Oettingen \& Gollwitzer, 2013), nos decidimos a hacer llegar el cuestionario a estos mediante las es- 
cuelas. Los cuestionarios que no fueron devueltos a las escuelas en el período establecido, que era de dos semanas, no formaron parte de la muestra, así como los que estaban incompletos (un $3 \%$ en este último caso). De este modo, se recopiló el total de la muestra, como ya hemos dicho formada por 711 alumnos.

\section{Análisis de datos}

Para los análisis estadísticos se utilizó el paquete IBM SPSS Statistics 17. En primer lugar, mediante la $t$ de Student se describieron y compararon las medias de las variables continuas, Mat, Len, TV y VJ, según el género del alumno. Después se correlacionaron estas cuatro variables cuantitativas entre sí, para comprobar si existía alguna relación entre ellas.

A continuación se calcularon cuatro ANOVAs de dos vías controlando para el género, uno con los minutos de TV como variable dependiente y otro con los de $\mathrm{VJ}$, ambos con las notas de Mat y Len dicotómicas-suspenso y aprobado- como variables independientes. Se desestimó la posibilidad de aplicar un ANCOVA con las cuatro variables a la vez porque para utilizar correctamente este procedimiento estadístico, las variables tomadas como dependientes, TV y VJ, no deberían estar relacionadas y, en nuestro caso, la correlación calculada anteriormente había demostrado una relación positiva y significativa entre ambas variables.

\section{Resultados.}

A continuación, en la Tabla 1 se muestra la descripción de las variables continuas y su comparación entre géneros con la $t$ de Student.

Como puede observarse en la Tabla 1 no hay diferencias entre niños y niñas en las notas de matemáticas ni en el tiempo dedicado a mirar la televisión. En cambio, sí hay diferencias significativas en las notas de lengua, que son más altas para las niñas, y en el tiempo dedicado a los videojuegos, en este caso con valores significativamente más altos para los alumnos de género masculino.

También hay que hacer notar que, de acuerdo a los datos presentados en la Tabla 1, los niños y niñas de educación primaria obtienen notas significativamente más altas en matemáticas que en lengua, pues la comparación de medias con datos apareados arroja una $t=4.48$, con una $p=.00$. Resulta igualmente interesante destacar que actualmente los alumnos y alumnas de primaria pasan significativamente más tiempo ante el televisor que jugando con pantallas, ya que la mis-

\begin{tabular}{|c|c|c|c|c|}
\hline VARIABLES CONTINUAS & GÉNERO & MEDIAS & $t$ & $p$ \\
\hline \multirow{2}{*}{ Notas de matemáticas } & Niños & 7.28 & \multirow{2}{*}{.43} & \multirow{2}{*}{.67} \\
\hline & Niñas & 7.34 & & \\
\hline \multirow{2}{*}{ Notas de lengua } & Niños & 6.75 & \multirow{2}{*}{3.73} & \multirow{2}{*}{.00} \\
\hline & Niñas & 7.32 & & \\
\hline \multirow{2}{*}{ Minutos a la semana de TV } & Niños & 471 & \multirow{2}{*}{1.20} & \multirow{2}{*}{.23} \\
\hline & Niñas & 444 & & \\
\hline \multirow{2}{*}{ Minutos a la semana de VJ } & Niños & 263 & \multirow{2}{*}{2.00} & \multirow{2}{*}{.04} \\
\hline & Niñas & 226 & & \\
\hline
\end{tabular}

Tabla 1. Comparación de medias de las variables cuantitativas en función del género de los alumnos de primaria $(n=711)$. 


\begin{tabular}{|c|c|c|c|}
\cline { 2 - 4 } \multicolumn{1}{c|}{} & MATEMÀTICAS & LENGUA & TV \\
\hline \multirow{2}{*}{ Len } & $\mathbf{. 7 3}$ & & \\
\hline \multirow{2}{*}{ TV } & $(.00)$ & & \\
& -.07 & -.05 & $(.20)$ \\
\hline \multirow{2}{*}{ VJ } & $(.06)$ & -.08 & .37 \\
& -.06 & $(.03)$ & $(.00)$ \\
\hline
\end{tabular}

Tabla 2. Correlaciones de Pearson entre las notas de lengua y matemáticas y el tiempo de televisión y videojuegos de los alumnos de primaria $(n=711)$. Se han escrito en negrita las correlaciones significativas.

ma prueba obtiene una $t=18.60$, con una $p=$ .00 .

A continuación se calcularon las correlaciones de Pearson entre las cuatro variables continuas. Los resultados de este análisis pueden observarse en la Tabla 2.
Tal como muestra la Tabla 2, las notas de lengua y matemáticas correlacionan positivamente y también el tiempo de televisión con el de videojuegos. Además, y éste es probablemente el dato más novedoso de esta tabla, se observa una correlación baja pero sig-

\begin{tabular}{|c|c|c|c|}
\hline ASIGNATURA & GÉNERO & $\begin{array}{l}\text { TIEMPO DE TV } \\
\text { Media (desviación) }\end{array}$ & $n$ \\
\hline Mat suspendidas & $\begin{array}{c}\text { Masculino } \\
\text { Femenino } \\
\text { Total }\end{array}$ & $\begin{array}{l}549(345) \\
518(331) \\
535(336) \\
\end{array}$ & $\begin{array}{l}32 \\
26 \\
58 \\
\end{array}$ \\
\hline Mat aprobadas & $\begin{array}{l}\text { Masculino } \\
\text { Femenino } \\
\text { Total }\end{array}$ & $\begin{array}{l}462(269) \\
439(304) \\
451(287)\end{array}$ & $\begin{array}{l}327 \\
326 \\
653\end{array}$ \\
\hline Len suspendida & $\begin{array}{l}\text { Masculino } \\
\text { Femenino } \\
\text { Total }\end{array}$ & $\begin{array}{l}536(296) \\
476(354) \\
508(323)\end{array}$ & $\begin{array}{l}29 \\
25 \\
54\end{array}$ \\
\hline Len aprobada & $\begin{array}{c}\text { Masculino } \\
\text { Femenino } \\
\text { Total }\end{array}$ & $\begin{array}{l}465(275) \\
442(303) \\
453(289)\end{array}$ & $\begin{array}{l}330 \\
327 \\
657 \\
\end{array}$ \\
\hline ASIGNATURA & GENERO & $\begin{array}{c}\text { TIEMPO VJ } \\
\text { Media (desviación) }\end{array}$ & $n$ \\
\hline Mat suspendidas & $\begin{array}{l}\text { Masculino } \\
\text { Femenino } \\
\text { Total }\end{array}$ & $\begin{array}{l}273(233) \\
183(153) \\
233(204)\end{array}$ & $\begin{array}{l}32 \\
26 \\
58\end{array}$ \\
\hline Mat aprobadas & $\begin{array}{l}\text { Masculino } \\
\text { Femenino } \\
\text { Total }\end{array}$ & $\begin{array}{l}262(234) \\
229(273) \\
246(254)\end{array}$ & $\begin{array}{l}327 \\
326 \\
653\end{array}$ \\
\hline Len suspendida & $\begin{array}{l}\text { Masculino } \\
\text { Femenino } \\
\text { Total }\end{array}$ & $\begin{array}{l}312(247) \\
314(388) \\
313(317)\end{array}$ & $\begin{array}{l}29 \\
25 \\
54\end{array}$ \\
\hline Len aprobada & $\begin{array}{l}\text { Masculino } \\
\text { Femenino } \\
\text { Total }\end{array}$ & $\begin{array}{l}259(232) \\
219(254) \\
239(244)\end{array}$ & $\begin{array}{l}330 \\
327 \\
657\end{array}$ \\
\hline
\end{tabular}

Tabla 3. Tiempo de televisión y de videojuegos en minutos a la semana (redondeados a la unidad y con la desviación típica entre paréntesis) según las notas en lengua y matemáticas y el género de los alumnos de educación primaria $(n=711)$. 
nificativa y en este caso negativa entre la nota de lengua y el tiempo de videojuegos, mientras que el tiempo de televisión no parece estar relacionado con los resultados académicos de los alumnos de primaria en las dos asignaturas analizadas.

Finalmente, y a fin de profundizar en la relación entre las variables estudiadas se calcularon cuatro ANOVAs de dos vías entre grupos. Se tomaron como variables independientes dos grupos de notas, suspenso y aprobado en matemáticas y lengua, y también el género, masculino y femenino, y como variables dependientes los minutos de televisión y de videojuegos a la semana, respectivamente. Los descriptivos de estos análisis están recogidos en la Tabla 3.

Como puede observarse en la Tabla 3, el primer ANOVA analizó la relación entre el tiempo de televisión, el género y las notas de matemáticas. Para la interacción género-matemáticas esta prueba obtuvo una $F(1.707)$ $=.01, p=.93$, eta parcial $=.00$, que pone en evidencia la ausencia de relación entre las variables estudiadas. De acuerdo con este resultado, esta prueba también destaca que sin tener en cuenta las notas de matemáticas, tampoco se observan diferencias entre niños y niñas en el tiempo de televisión, ya que $\mathrm{F}$ $(1.707)=.48, p=.49$, eta parcial $=.01$. No obstante, dejando aparte el género de los alumnos, el resultado del análisis para las horas de televisión y las notas académicas de matemáticas es de $F(1.707)=4.22, p=.04$, eta parcial $=.01$, lo que indica que con independencia del género, los alumnos de primaria que suspenden matemáticas dedican más tiempo semanal a la televisión que los que aprueban esta asignatura. Estos datos quedan reflejados en la Figura 1.

El segundo ANOVA estudió la relación entre el tiempo de televisión, el género y las notas de lengua. Este análisis no ha dado ninguna relación significativa. La interacción entre las tres variables da una $F(1.707)=.19$, $p=.66$, eta parcial $=.00$; la conexión entre las

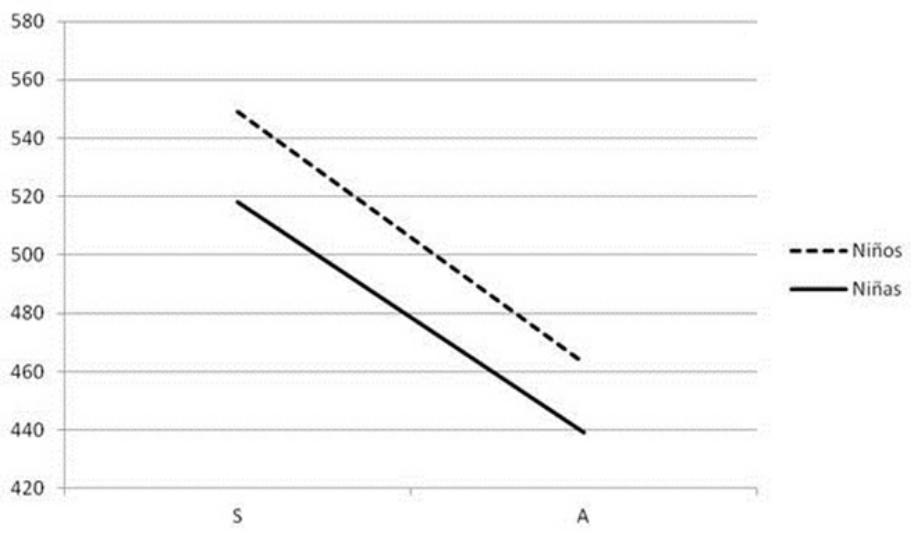

Figura 1. Representación gráfica de los valores medios de minutos de televisión a la semana para alumnos de primaria según la nota de matemáticas, S suspenso y A aprobado, y el género $(n=711)$.

Píxel-Bit. Revista de Medios y Educación. No 46. Enero 2015. ISSN: 1133-8482. e-ISSN: 2171-7966. doi: http://dx.doi.org/10.12795/pixelbit.2015.i46.02 


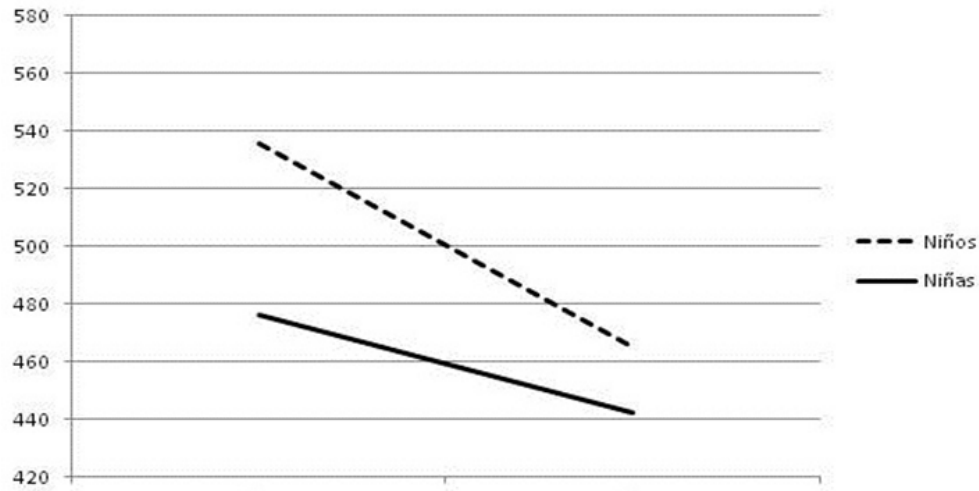

Figura 2.Representación gráfica de los valores Ânedios de minutos de televisión a la semana para alumnos de primaria según la nota de lengua, $S$ suspenso y A aprobado, y el género $(n=711)$.

horas de televisión y las notas de lengua es $\operatorname{de} F(1.707)=1.59, p=.21$, eta parcial $=.00 ; \mathrm{y}$ la relación entre el tiempo de TV y el género, de $F(1.707)=.99, p=.32$, eta parcial $=.00$. Así pues, estos resultados indican que no hay relación entre el tiempo de TV, las calificaciones en la asignatura de lengua y el género de los alumnos de primaria. La descripción de los datos está representada gráficamente en la Figura 2.

El tercer ANOVA se calculó para comprobar la relación entre el tiempo dedicado a los videojuegos, el género y las notas de matemáticas. A diferencia del primer ANOVA, de-

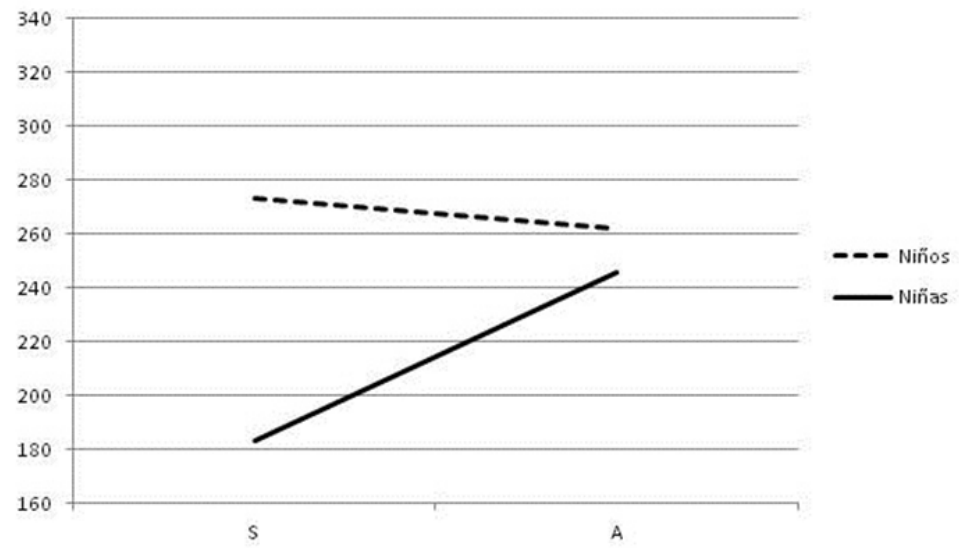

Figura 3. Representación gráfica de los valores medios de minutos de juegos con pantallas a la semana para alumnos de primaria según la nota de matemáticas, $S$ suspenso y A aprobado, y el género $(n=711)$.

Píxel-Bit. Revista de Medios y Educación. No 46. Enero 2015. ISSN: 1133-8482. e-ISSN: 2171-7966. doi: http://dx.doi.org/10.12795/pixelbit.2015.i46.02 


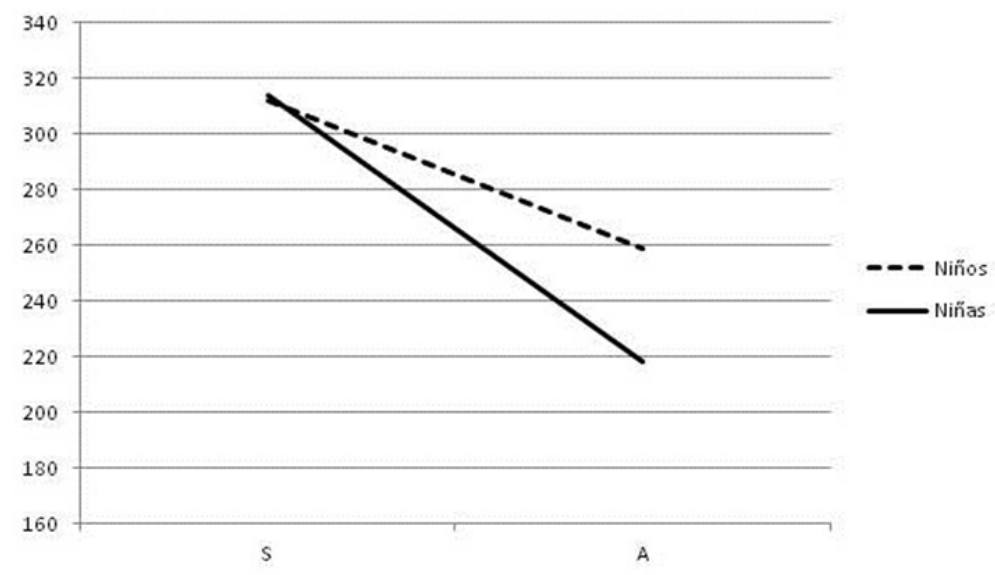

Figura 4. Representación gráfica de los valores medios de minutos de videojuegos a la semana para alumnos de primaria según la nota de lengua, S suspenso y A aprobado, y el género $(n=711)$.

dicado a la TV, en este caso, con VJ, el efecto de interacción entre género y matemáticas no dio ningún resultado significativo, $F(1.707)$ $=.66, p=.42$, eta parcial $=.00$. Tampoco se observaron diferencias importantes entre niños y niñas, $F(1.707)=.3 .17, p=.07$, eta parcial $=.00$, aunque quizás sería conveniente repetir este último análisis con una muestra más amplia de alumnos con «matemáticas suspendidas» ya que los valores, aunque no significativos, son muy ajustados. Tampoco la relación entre las notas de matemáticas y el tiempo de VJ arrojó resultados significativos, $F(1.707)=26, p=.61$, eta parcial $=.00$. Una representación gráfica de estos resultados se puede observar en la Figura 3.

Para acabar, el ANOVA de dos vías entre el tiempo de videojuegos y las notas de lengua, con control para el género, arrojó un efecto de interacción de $F(1.707)=.35, p=.55$, eta parcial $=.00$, indicando que no hay relación entre las tres variables tomadas conjuntamen- te. Las diferencias entre niños y niñas tampoco son significativas, $F(1.707)=.29, p=$ .59 , eta parcial $=.00$. En cambio, el análisis revela un efecto estadísticamente significativo para las notas de lengua y el tiempo de televisión, pues $F(1.707)=4.43, p=.04$, eta parcial $=.01$. Este resultado indica que los alumnos -niños y niñas- de educación primaria que suspenden la lengua dedican más tiempo a los juegos con pantallas que todos los demás alumnos y alumnas que aprueban esta asignatura. Estos datos están representados en el gráfico de la Figura 4.

Las Figuras 1 y 4, justifican la realización de los análisis por separado. Está claro que el género del alumno influye tanto en los resultados académicos como en el tiempo pasado ante el televisor y jugando con pantallas, pero esta influencia no se produce de la misma manera sino con las salvedades que para cada ANOVA hemos explicado. 


\section{Discusión y conclusiones.}

De los resultados hallados en este estudio, podemos concluir que los alumnos de la muestra pasan más tiempo viendo la televisión que jugando con los videojuegos, lo que es totalmente congruente con resultados hallados en los estudios de Gonzalez, Rodiño, Gorís y Carballo (2008). Al analizar detalladamente las variables videojuegos y televisión, observamos diversos resultados. Tanto en niños como en niñas que ven excesivas horas la televisión, destaca el hecho de que sea el rendimiento en el área de matemáticas el más perjudicado, no siendo así para los niños que dedican más horas a los videojuegos. Estos resultados podrían estar en consonancia con aquellos que indican que el uso de videojuegos favorece la lógica, la espontaneidad, el razonamiento y la originalidad (Marks, 1985; Gagnon; 1985; Silvern, 1986; Greenfield, 1994; Ricci, 1994; Marquès, 2000; Rodríguez, 2002). Como ya hemos comentado, estos autores defienden que su uso representa un importante potencial educativo ya que estimulan la capacidad lógica, el desarrollo de estrategias encaminadas a la resolución de problemas, la perseverancia y la tolerancia al fracaso. Al ayudar también a desarrollar la coordinación viso-manual, los videojuegos constituyen una interesante introducción al uso de la informática y las nuevas tecnologías.

En lo que concierne el área de lengua, los alumnos que cosechan mayor número de suspensos son los que dedican a los videojuegos más tiempo en comparación a sus compañeros que aprueban esta materia.Una posible explicación a este resultado podría ser que los niños que pasan muchas horas delante de este tipo de pantallas desarrollan insufi- cientemente las competencias lingüísticas al obtener básicamente material visual.

Que los niños dediquen más tiempo a jugar con videojuegos que las niñas, cosa que les dificultaría el desarrollo del área de lengua, parece coherente con los datos obtenidos en los estudios de Hayes (2007) donde se destaca la mayor competencia de éstos, y una actitud más positiva hacia el uso de videojuegos que las niñas. A ello cabría añadir el hecho de que los videojuegos suelen estar diseñados para niños puesto que los papeles femeninos pocas veces tienen el rol protagonista.

Los resultados hallados en nuestro estudio muestran algunos efectos de la paradoja que, en la actualidad, se vive en el mundo educativo: nuestros estudiantes están básicamente orientados al mundo de la imagen, pero una mayoría de profesores proceden del mundo del discurso al que, por otra parte, no habría que renunciar. Cómo conseguir la compatibilidad entre ambos mundos constituye un auténtico reto en la sociedad actual.

Finalmente, queremos indicar algunas limitaciones de este trabajo. Por una parte, utilizar una encuesta general para indagar sobre los usos de los videojuegos y horas de televisión, y el rendimiento académico de los estudiantes, resulta interesante y operativo para poder trabajar con muestras grandes, pero este tipo de metodología, no permite profundizar en los temas. En este sentido, el empleo de entrevistas que facilita un acceso más amplio y directo a la información objeto de estudio, podría aportar no sólo datos sobre la magnitud de las relaciones estudiadas, sino elementos explicativos y justificativos de las mismas. A otro nivel, sería interesante analizar cómo cambia el patrón tecnológico en función del desarrollo de los alumnos. Es decir, si hay una diversificación o concentración 
del uso de videojuegos y/o televisión a medida que los alumnos crecen. Contemplamos la posibilidad de ampliar la muestra de estudio con población internacional, para poder observar si es posible una generalización de los datos obtenidos, así como de analizar la posible existencia de diferencias según el nivel sociocultural y demográfico de los sujetos estudiados.

\section{Referencias bibliográficas.}

Aguilar, J., Cumbá, C., Cortés, A., Collado, A.M., García, R. \& Pérez, D. (2010). Habits or inappropriate behaviors and poor academic results in students of secondary school. $R e$ vista Cubana de higiene y epidemiología,48 (3), 280-290.

Álvarez, J.M. \& López, J. (2007). Alternativas de política cultural. Las industrias culturales en las redes digitales. Barcelona: Gedisa.

Bailey, K., West, R. \& Anderson, C.A. (2010). A negative association between video game experience and proactive cognitive control. Psychophysiology, 47 (1), 34-42.doi: 10.1111/j.1469-8986.2009.00925.x

Bode, H. \& Hirner, V. (2013). Children with learning disabilities and handicaps in inclusive schools or in special schools? The view of parents and professionals. KlinPadiatr, 225 (2), 57-63.doi:10.1055/s-0033-1333760

Bringas, C., Rodríguez, F. \& Herrero, F.J. (2008). Adaptación y motivación escolar: Análisis de la influencia del consumo de medios electrónicos de comunicación por adolescentes. Cuadernos de trabajo social, 21, 141-153.

Castells, P. \& Bofarull, I. (2002). Enganchados a las pantallas: Televisión, videojuegos, Internet y móviles. Barcelona: Planeta, S.A.
Clemente, M. \& Vidal, M.A. (1996). Violencia y televisión. Madrid: Noésis.

Donnerstein, E. (2004). Medios de comunicación. En J. Sanmartin (ed). El laberinto de la violencia: causas tipos y efectos. Barcelona: Ariel.

Etxeberria, F. (1999). Videojuegos y educación. En Etxeberria, F. (Coord). La Educación en Telépolis. Donostia: Editorial Ibaeta.

Etxeberria, F. (2008). Videojuegos consumo y educación. Teoría de la Educación: Educación y Cultura en la Sociedad de la Información, 9 (3), 1-28.

Ferguson, C.J. (2011). The influence of television and video game use on attention and school problems: A multivariate analysis with other risk factors controlled. Journal of Psychiatric Research, 45 (6),808-813.doi: 10.1016/j.jpsychires.2010.11.010

Gagnon, D. (1985). Videogames and Spatial Skills: An Exploratory Study. Educational Communication and Technology, 33 (4), 263275.doi: 10.1007/BF02769363

Garrido, M. (2004). Violencia, televisión y publicidad. Sevilla: Alfar.

Gawrilow, C., Morgenroth, K., Schultz, R., Oettingen, G. \& Gollwitzer, P.M. (2013). Inducing self-regulation of goal pursuit increases self-discipline in schoolchildren at risk and not at risk for ADHD. Motivation and Emotion, 37, 134-145.

Gonzalez, C., Rodiño, S., Gorís, A. \& Carballo, A. (2008). Consumo de medios de comunicación en una población infantojuvenil. Revista Pediatría de Atención Primaria, 10 (38), 53-67.

Green, C. Shawn \& Bavelier, Daphne (2003). Action video game modifies visual selective attention. Nature, 423, 534-537. doi:10.1038/ nature01647.

Greenfield, P. (1994). Cognitive socialization by computer games in two cultures: Inductive 
discovery or mastery of an iconic code? Journal of Applied Developmental Psychology, 15 (1), 59-85. doi:.org/10.1016/ 0193-3973(94)90006-X.

Gros, B. (2007). Videojuegos y aprendizaje. Barcelona: Graó.

Hayes, E. (2007). Gendered Identities at play: Case studies of two women playing Morrowind. Games and Culture, 2 (1), 23-48.

Kaiser Family Foundation (2003). Zero to Six: Media Use in the Lives of Infants, Toddlers, and Preschoolers.KFF. Menlo Park.

Llorca, M.A., Bueno, G.M., Villar, C. \& Díez, M.A. (2010).Frecuencia en el uso de videojuegos y rendimiento académico. II Congreso Internacional Comunicación 3.0 (4 y 5 de Octubre). Salamanca.

López, N., González, P. \& Medina, E. (2011). Jóvenes y televisión. Un cambio de hábitos. ZER, 16 (30), 97-113.

Mandinacht, E. (1987). Clarifying the «A» in CAI for learners of different abilities. Journal of Educational Computing Research,3 (1), 113-128.doi:10.2190/2V9MX43N-WJ2C-DG9N

Mark, A., Boyce, F. \& Janssen, I. (2006). Television viewing, computer use and total screen time in Canadian youth. Journal ListPaediatric Child Health, 11 (9), 595-599.

Marks, P. (1985). Mind and Media. The effects of Television, computers and videogames. London: William Collins Sons\& Co. Ltd.

Marquès, P. (2000). Videojuegos. Las claves del éxito. Cuadernos de Pedagogía, 291, 55-62.

McFarlane, A. \& Sakellariou, S. (2002). The role of ICT in science education. Cambridge Journal of Education, 32 (2), 219-232.doi: 10.1080/03057640220147568

Okagaki, L. \& Frensch, P. (1994). Effects of video game playing on measures of spatial perfomance: gender effects in late adolescence. Journal of Applied Development Psychology, 15(1), 3358.doi.org/10.1016/0193-3973(94)90005-1

Pagani, L.S., Fitzpatrick, M.A., Barnett, T.A. \& Dubow, E. (2010).Prospective associations between early childhood television exposure and academic, psychosocial, and physical well-being by middle childhood. Archives of Pediatrics and Adolescent Medicine, 164 (5), 425-431. doi:10.1001/archpediatrics.2010.50

Ricci, K.E. (1994). The use of computerbased videogames in knowledge acquisition and retention. Journal of InteractiveInstructionDevelopment, 7 (1), 17-22.doi:10.1207/s15327876mp0804_3

Rodríguez, A. (2005). Los efectos de la televisión en niños y adolescentes. Comunicar: Revista científica iberoamericana de comunicación y educación,25 (2), 1-14.

Rodríguez, E. (2002). Jóvenes y Videojuegos: Espacio, significación y conflictos. Madrid: Ministerio de Trabajo y Asuntos Sociales (INJUVE).

Roschelle, J.M., Pea, R.D., Hoadley, C.M., Gordin, D.N. \& Means, B.M. (2000). Changinghow and what children learn in school with computer-based technologies. TheFuture of Children, 10 (2), 76-101.

Sedeño, A. (2010). Videojuegos como dispositivos culturales: las competencias espaciales en educación. Comunicar, 17 (34), 183189.doi:10.3916/C34-2010-03-018

Silvern, S.B. (1986). Classroom Use of Video Games. Educational Research Quarterly, $10(1), 10-16$.

Singh, A. \& Gaurav, S. (2013).Television Exposure and Academic Skills of Children: New Findings from India. Journal of Communication Technology and Human Behaviors, 1, 1-24.doi:10.7726/jcthb. 2013.1001 
Trick, Lana M., Jaspers-Fayer, Fem \& Sethi, Lana M. (2005). Multiple-object tracking in children: The Catch the Spies task. Cognitive Development, 20 (3), 373-387. doi:10.1016/ j.cogdev.2005.05.009.

Ulpiano, V. (1995). La televisión y el rendimiento escolar de los alumnos de Enseñanza General Básica. Revista Interuniversitaria de Formación del Profesorado, 22 (9), 125-138.

Vallejos, M. \& Capa, W. (2010). Video juegos: adicción y factores predictores. $A v$. Psicol., 18 (1), 103-110.

Villadangos, S. \& Labrador, F. (2009). Menores y nuevas tecnologías ¿uso o abuso?.Annuary of Clinical and HealthPsychology, 5, 75-83.

White, B. (1984). Designing computer games to help physics students understanding Newton's laes of motion. Cognition and Instruction, 1 (1), 69108.doi:10.1207/s1532690xci0101_4

Fecha de recepción: 14-03-2014

Fecha de evaluación: 14-05-2014

Fecha de aceptación: 06-06-2014

Píxel-Bit. Revista de Medios y Educación. No 46. Enero 2015. ISSN: 1133-8482. e-ISSN: 2171-7966. doi: http://dx.doi.org/10.12795/pixelbit.2015.i46.02 